\title{
The relation between vitamin D status and anemia in patients with end stage renal disease on regular hemodialysis
}

\section{Essam Nour el Din Afifi ${ }^{1}$, Ahmed Mohamed Tawfik ${ }^{1}$, Essam Eldin Rashad Saeed Abdulkhalek ${ }^{2}$, Lina Essam Khedr ${ }^{* * \mathbb{D}}$}

\begin{abstract}
Introduction: Anemia is a common complication in end-stage renal disease (ESRD) patients on regular hemodialysis (HD). There has been a lot of interest recently in the non-classical effects of $25(\mathrm{OH})$ vitamin $\mathrm{D}$ (calcidiol), including its association with erythropoiesis and anemia pathogenesis.

Objectives: To study the relation between anemia and vitamin D status in patients on regular HD.

Patient and Methods: This study is a cross-sectional study that included 90 patients on regular HD. Vitamin D status was classified into deficient $(<20 \mathrm{ng} / \mathrm{mL})$, insufficient $(20-30 \mathrm{ng} / \mathrm{mL})$ and sufficient $(>30 \mathrm{ng} / \mathrm{mL})$.

Results: The level of vitamin D measured in the patients ranged between 3.5 to $66 \mathrm{ng} / \mathrm{mL}$ with median of $16.35 \mathrm{ng} / \mathrm{mL}$. There were statistically significant positive correlations between vitamin D levels and the level of hemoglobin $(P<0.001)$, serum calcium levels $(P<0.001)$ and serum PO4 levels $(P=0.023)$. Higher hemoglobin levels were statistically related to both higher vitamin $\mathrm{D}$ values $(P<0.001)$ and higher serum calcium concentration $(P<0.001)$. Meanwhile, a significant negative correlation was found between hemoglobin levels and serum PTH values $(P<0.001)$.

Conclusion: There was a significant association between the status of vitamin D and the level of hemoglobin in dialysis population who were studied, independent from iron status. other associations with hemoglobin levels included PTH level and calcium.

Keywords: Vitamin D, Calcidiol, Anemia, Dialysis, End-stage renal disease

Citation: Nour el Din Afifi E, Tawfik AM, Abdulkhalek EERS, Essam Khedr L. The relation between vitamin D status and anemia in patients with end stage renal disease on regular hemodialysis. J Renal Endocrinol. 2021;7:e15. doi: 10.34172/jre.2021.15.

Copyright (C) 2021 The Author(s); Published by Nickan Research Institute. This is an open-access article distributed under the terms of the Creative Commons Attribution License (http://creativecommons.org/licenses/by/4.0), which permits unrestricted use, distribution, and reproduction in any medium, provided the original work is properly cited.
\end{abstract}

\section{Introduction}

Anemia is a complication that is commonly encountered when caring for patients with chronic kidney disease (CKD). Moreover, the severity of the anemia worsens as the glomerular filtration rate (GFR) declines, with a prevalence reaching approximately $90 \%$ in patients with a GFR less than $30 \mathrm{~mL} / \mathrm{min}(1)$.

Previous studies have found anemia to be associated with significant morbidity and mortality in end-stage renal disease (ESRD) patients on hemodialysis (HD). Anemia leads to progression of left ventricular hypertrophy with increased risk of myocardial infarction and heart failure $(2,3)$. Anemia has also been associated with depression, cerebrovascular stroke, and fatigue and reduced exercise tolerance (4).

The pathogenesis of anemia in CKD is multifactorial. The fall in GFR is typically accompanied by a decrease in erythropoietin production. However, several other factors contribute including absolute and functional iron deficiency, folate and vitamin $\mathrm{B}_{12}$ deficiencies and suppression of erythropoiesis by uremia (5).
Among patients with CKD or those on dialysis, vitamin $\mathrm{D}$ deficiency and insufficiency are increasingly common (6). Diet restrictions and decreased sunlight exposure are factors that affect vitamin D levels in dialysis patients. Del Valle et al reported inadequate sunlight exposure in approximately $84 \%$ of $\mathrm{HD}$ patients with vitamin $\mathrm{D}$ deficiency (7). Furthermore, uremia has a negative effect on the activation of plasma vitamin $\mathrm{D}$ in response to ultraviolet B (UVB) irradiation (8). One of the most common cutaneous manifestations in patients undergoing HD is hyperpigmentation, which too can impair the activation of vitamin D (9). Understanding vitamin D physiology has sparked interest in recent studies searching for other functions beyond its role in mineral and bone metabolism (10). It has been proven that activation of vitamin $\mathrm{D}$ can occur in several tissues, in addition to the kidneys (11). Several studies have shown that an increased risk of cardiovascular, musculoskeletal and autoimmune diseases, cancer and increased risk of infections can be associated with vitamin D deficiency $(12,13)$. The study to evaluate early kidney disease and the third national health 
Implication for health policy/practice/research/ medical education

In a cross-sectional study that included 90 patients on regular hemodialysis, we found a statistically significant positive correlation between vitamin D levels and the level of hemoglobin.

and nutrition examination survey showed that vitamin D deficiency was significantly and independently associated with anemia in patients with CKD who do not require dialysis $(14,15)$.

\section{Objectives}

This study aimed to evaluate the relation between anemia and vitamin $\mathrm{D}$ status in patients on regular $\mathrm{HD}$

\section{Patients and Methods}

\section{Study design}

This is a cross-sectional study involving 90 patients in El Agouza hospital, Giza, Egypt, with ESRD on regular HD treatment for more than six months with age over 18 years. This study excluded patients with hepatitis B or hepatitis C infection, patients with malignancies, patient with hemolytic anemia, patients with malabsorption syndromes (Celiac disease and inflammatory bowel disease), patients with active or chronic infections, patients with active bleeding and renal transplant recipients. All patients were on fixed dose of erythropoietin 4000 units twice weekly, except those with hemoglobin more than or equal to 11 g/dL. The National Kidney Foundation-Kidney Disease Outcomes Quality Initiative (NKF-KDOQI) guidelines (2012) recommended that $\mathrm{Hb}$ targets should be in the range of 10.0-11.5 g/dL, regardless of whether the patients were receiving dialysis (16). Therefore, we defined anemia as an $\mathrm{Hb}$ level of $<11 \mathrm{~g} / \mathrm{dL}$.

All patients were subjected to full history and physical examination with emphasis on demographic data age, gender, duration of $\mathrm{HD}$, cause of renal failure, comorbidities, and medications. Laboratory tests included serum calcium, phosphorus, 25(OH) D and intact parathyroid hormone (iPTH) levels, hemoglobin level, ferritin level, serum iron, total iron binding capacity (TIBC) and calculation of transferrin saturation (TSAT). Serum 25(OH) vitamin D level was measured by ELISA (Cal biotech. USA, REF: VD2208), serum samples were collected and centrifuged and separated as soon as possible, stored at $-20^{\circ} \mathrm{C}$, all specimens were allowed to come to room temperature before use. Vitamin $\mathrm{D}$ deficiency was defined as serum levels $<20 \mathrm{ng} / \mathrm{dL}$, insufficiency 20-30 $\mathrm{ng} / \mathrm{dL}$ and sufficiency $>30 \mathrm{ng} / \mathrm{dL}$.

\section{Ethical issues}

The research followed the tenets of the Declaration of Helsinki. This study was approved by Ain Shams University ethical committee (Ref\# FWA000017585).
Informed consent was taken from all participants.

\section{Statistical analysis}

IBM SPSS statistics (V. 25.0, IBM Corp., USA, 2017-2018) was used for data analysis. Data were expressed as median and percentiles for quantitative non-parametric measures. The following tests were conducted: (a) Comparison between two independent groups for non-parametric data using Wilcoxon rank sum test; (b) Comparison between more than 2 patient groups for non-parametric data using Kruskal-Wallis test; and (c) Ranked Spearman's correlation test to study the possible association between each two variables among each group for non-parametric data. The probability of error at 0.05 was considered significant, while at 0.01 and 0.001 are highly significant.

\section{Results}

This study recruited 90 patients with ESRD, maintained on regular HD in El Agouza hospital HD unit. Table 1 shows the demographics of the study population with the mean age of $51.13 \pm 10.44$ years consisted of 68 males and 22 females with median duration of HD of 9.50 (6-2) years. Around 32 (35.6\%) of patients were diabetic while 86 (95.6\%) of them were hypertensive. As regards the etiology of ESRD the majority of the patients listed hypertension and diabetes $37(41.1 \%)$ and $32(35.6 \%)$ patients respectively.

The level of vitamin $\mathrm{D}$ measured in the patients ranged between 3.5 to $66 \mathrm{ng} / \mathrm{mL}$ with median of $16.35 \mathrm{ng} / \mathrm{mL}$ (8$34 \mathrm{ng} / \mathrm{mL}$ ). About 24 patients had vitamin D deficiency (vitamin D levels <20 ng/dL) (Table 2). Accordingly, patients with insufficient levels (vitamin D levels 20-

Table 1. Demographic data of the study population

\begin{tabular}{llc}
\hline & & $\mathrm{N}=\mathbf{9 0}$ \\
\hline \multirow{2}{*}{ Gender } & Female & $22(24.4 \%)$ \\
& Male & $68(75.6 \%)$ \\
\hline \multirow{2}{*}{ Age $(\mathrm{y})$} & Mean \pm SD & $51.13 \pm 10.44$ \\
Dialysis duration (y) & Median (IQR) & $24-66$ \\
& Range & $9.50(6-12)$ \\
Diabetic & No & $1-21$ \\
& Yes & $58(64.4 \%)$ \\
HTN & No & $32(35.6 \%)$ \\
& Yes & $4(4.4 \%)$ \\
& Hypertension & $86(95.6 \%)$ \\
& Diabetic nephropathy & $37(41.1 \%)$ \\
& Lupus nephritis & $32(35.6 \%)$ \\
& Chronic glomerulonephritis & $4(4.4 \%)$ \\
& Chronic tubulointerstitial nephritis & $4(4.4 \%)$ \\
& ADPKD & $2(2.2 \%)$ \\
& Hereditary nephritis & $1(1.1 \%)$ \\
& Amyloidosis & $1(1.1 \%)$ \\
& Unknown & $1(1.1 \%)$ \\
& & $8(8.9 \%)$ \\
\hline
\end{tabular}

ESRD, end-stage renal disease; ADPKD, autosomal dominant polycystic kidney disease. 
Table 2. Classification of the patients according to vitamin D3 levels (ng/dL)

\begin{tabular}{lc}
\hline Vitamin D3 & No. $(\%)$ \\
\hline Deficient & $24(26.7 \%)$ \\
Insufficient & $32(35.6 \%)$ \\
Sufficient & $34(37.8 \%)$ \\
Median (IQR) & $16.35(8-34)$ \\
Range & $3.5-66.3$ \\
\hline
\end{tabular}

$30 \mathrm{ng} / \mathrm{dL}$ ) were 32 individuals, while patients with sufficient ( $>30 \mathrm{ng} / \mathrm{dL}$ ) levels were 34 cases. Table 3 shows no statistically significant relations between vitamin $\mathrm{D}$ levels and the gender of the patients $(P=0.536)$, their age $(P=0.973)$, duration of HD $(P=0.600)$, diabetes mellitus $(P=0.064)$ and hypertension $(P=0.249)$.

There were statistically significant positive correlations between vitamin $\mathrm{D}$ levels and the value of hemoglobin $(P<0.001)$, serum calcium levels $(P<0.001)$ and serum PO4 levels $(P=0.023)$. However, significant negative correlations of vitamin D levels with serum iPTH levels $(P<0.001)$, serum iron $(P=0.019)$ and TSAT $(P=0.012)$ were detected. There was no statistically significant relation between vitamin $\mathrm{D}$ levels and type of dialysis access $(P=0.437)$, TIBC $(P=0.193)$, serum ferritin levels $(P=0.125)$ or serum albumin concentration $(P=0.541)$ (Table 4).

In Table 5, using Spearman's correlation coefficient, highly significant positive correlation between vitamin D3 levels and both hemoglobin levels $(P<0.001)$ and serum calcium levels $(P<0.001)$ were detected. Meanwhile, there was highly significant negative correlation between vitamin D3 levels and serum iPTH (intact PTH) levels $P<0.001)$ and TSAT $(P=0.003)$.

There were no significant correlations between vitamin D3 levels in all three groups of patients and age of the patients, duration of HD, serum phosphorus levels, serum iron levels, TIBC, serum ferritin levels or serum albumin levels (Table 6).

Table 7 shows highly significant positive relation between hemoglobin levels of all patients and their vitamin D3 levels $(P<0.001)$. Meanwhile, no significant relationship between hemoglobin level and presence of diabetes $(P=0.533)$ or hypertension $(P=0.748)$, etiology of ESRD $(P=0.302)$ and type of dialysis access $(P=0.960)$ was detected.

Additionally, highly significant positive correlations between plasma hemoglobin concentration with both vitamin D3 $(P<0.001)$ and serum calcium levels $(P<0.001)$ were seen. Meanwhile, a highly significant negative correlation between hemoglobin levels and serum iPTH levels was seen $(P<0.001)$.

There were no statistically significant correlations between hemoglobin levels and age of the patients, duration of $\mathrm{HD}$, serum phosphorus levels, serum iron, TIBC, serum ferritin levels, TSAT or serum albumin levels (Table 8).

\section{Discussion}

Observational studies have reported that vitamin D deficiency worsens with progression of CKD stage (17).

Table 3. Vitamin D levels in the 3 groups and demographic data

\begin{tabular}{|c|c|c|c|c|c|c|c|}
\hline & \multicolumn{7}{|c|}{ Vitamin D3 levels (ng/dL) } \\
\hline & & $\begin{array}{c}\text { Deficient } \\
n=24\end{array}$ & $\begin{array}{c}\text { Insufficient } \\
\mathbf{n}=\mathbf{3 2}\end{array}$ & $\begin{array}{c}\text { Sufficient } \\
n=34\end{array}$ & Test value & $P$ value & Sig. \\
\hline \multirow{2}{*}{ Gender } & Female & $5(20.8 \%)$ & $10(31.2 \%)$ & $7(20.6 \%)$ & \multirow{2}{*}{1.246} & \multirow{2}{*}{0.536} & \multirow{2}{*}{ NS } \\
\hline & Male & $19(79.2 \%)$ & $22(68.8 \%)$ & $27(79.4 \%)$ & & & \\
\hline \multirow{2}{*}{ Age } & Mean \pm SD & $51.33 \pm 10.18$ & $50.78 \pm 10.61$ & $51.32 \pm 10.76$ & \multirow{2}{*}{0.028} & \multirow{2}{*}{0.973} & \multirow{2}{*}{ NS } \\
\hline & Range & 24-63 & 24-65 & $26-66$ & & & \\
\hline \multirow{2}{*}{ Dialysis duration } & Median (IQR) & $9.5(6.5-11.5)$ & $10(7-12)$ & $8(5-11)$ & \multirow{2}{*}{1.021} & \multirow{2}{*}{0.600} & \multirow{2}{*}{ NS } \\
\hline & Range & 2-18 & $1-21$ & $1-17$ & & & \\
\hline \multirow{2}{*}{ Diabetic } & No & $14(58.3 \%)$ & $17(53.1 \%)$ & 27 (79.4\%) & \multirow{2}{*}{5.505} & \multirow{2}{*}{0.064} & \multirow{2}{*}{ NS } \\
\hline & Yes & $10(41.7 \%)$ & $15(46.9 \%)$ & $7(20.6 \%)$ & & & \\
\hline \multirow{2}{*}{ HTN } & No & $0(0.0 \%)$ & $1(3.1 \%)$ & $3(8.8 \%)$ & \multirow{2}{*}{2.783} & \multirow{2}{*}{0.249} & \multirow{2}{*}{ NS } \\
\hline & Yes & $24(100.0 \%)$ & $31(96.9 \%)$ & $31(91.2 \%)$ & & & \\
\hline \multirow{9}{*}{ Etiology of ESRD } & Hypertension & $11(45.8 \%)$ & $7(21.9 \%)$ & $19(55.9 \%)$ & \multirow{9}{*}{27.089} & \multirow{9}{*}{0.041} & \multirow{9}{*}{$\mathrm{S}$} \\
\hline & Diabetic nephropathy & $10(41.7 \%)$ & $15(46.9 \%)$ & $7(20.6 \%)$ & & & \\
\hline & Lupus nephritis & $1(4.2 \%)$ & $2(6.2 \%)$ & $1(2.9 \%)$ & & & \\
\hline & Chronic glomerulonephritis & $0(0.0 \%)$ & $0(0.0 \%)$ & $4(11.8 \%)$ & & & \\
\hline & Chronic tubulointerstitial nephritis & $0(0.0 \%)$ & $2(6.2 \%)$ & $0(0.0 \%)$ & & & \\
\hline & ADPKD & $0(0.0 \%)$ & $1(3.1 \%)$ & $0(0.0 \%)$ & & & \\
\hline & Hereditary nephritis & $0(0.0 \%)$ & $0(0.0 \%)$ & $1(2.9 \%)$ & & & \\
\hline & Amyloidosis & $0(0.0 \%)$ & $0(0.0 \%)$ & $1(2.9 \%)$ & & & \\
\hline & Unknown & $2(8.3 \%)$ & $5(15.6 \%)$ & $1(2.9 \%)$ & & & \\
\hline
\end{tabular}

ESRD, end-stage renal disease; ADPKD, autosomal dominant polycystic kidney disease.

$P$ value $<0.05$ : Significant; $P$-value $<0.01$ : Highly significant. 
Table 4. Vitamin D level in 3 groups in relation to clinical parameters including

\begin{tabular}{|c|c|c|c|c|c|c|c|}
\hline & & \multicolumn{3}{|c|}{ Vitamin D3 } & \multirow[b]{2}{*}{ Test value } & \multirow[b]{2}{*}{$P$ value } & \multirow[b]{2}{*}{ Sig } \\
\hline & & $\begin{array}{c}\text { Deficient } \\
n=24\end{array}$ & $\begin{array}{c}\text { Insufficient } \\
\mathrm{n}=32\end{array}$ & $\begin{array}{c}\text { Sufficient } \\
\mathrm{n}=34\end{array}$ & & & \\
\hline \multirow{3}{*}{ Dialysis access } & Arteriovenous fistula & $23(95.8 \%)$ & $29(90.6 \%)$ & $33(97.1 \%)$ & \multirow{3}{*}{$3.774^{\mathrm{a}}$} & \multirow{3}{*}{0.437} & \multirow{3}{*}{ NS } \\
\hline & Arteriovenous graft & $1(4.2 \%)$ & $1(3.1 \%)$ & $1(2.9 \%)$ & & & \\
\hline & Permanent HD catheter & $0(0.0 \%)$ & $2(6.2 \%)$ & $0(0.0 \%)$ & & & \\
\hline \multirow{2}{*}{ Serum calcium (mg/dL) } & Mean \pm SD & $8.57 \pm 1.07$ & $8.43 \pm 0.67$ & $9.32 \pm 0.68$ & \multirow{2}{*}{$11.646^{\mathrm{b}}$} & \multirow{2}{*}{0.000} & \multirow{2}{*}{$\mathrm{HS}$} \\
\hline & Range & $7.6-11.9$ & $6.9-9.7$ & $8.6-12$ & & & \\
\hline \multirow{2}{*}{ Serum phosphorus (mg/dL) } & Mean \pm SD & $4.63 \pm 0.81$ & $4.52 \pm 0.92$ & $5.32 \pm 1.67$ & \multirow{2}{*}{$3.935^{b}$} & \multirow{2}{*}{0.023} & \multirow{2}{*}{ S } \\
\hline & Range & $2.9-7.2$ & $2.6-8.1$ & $2.7-11$ & & & \\
\hline \multirow{2}{*}{ PTH (pg/mL) } & Median (IQR) & 669.5 (468.5-782.5) & $528.5(380-666.5)$ & $157.5(112-213)$ & \multirow{2}{*}{$50.646^{c}$} & \multirow{2}{*}{0.000} & \multirow{2}{*}{$\mathrm{HS}$} \\
\hline & Range & 230-1197 & $46.7-1107$ & $28-444$ & & & \\
\hline \multirow{2}{*}{ Hemoglobin (g/dL) } & Mean \pm SD & $8.73 \pm 0.83$ & $9.24 \pm 1.43$ & $11.24 \pm 1.83$ & \multirow{2}{*}{$24.660^{\mathrm{b}}$} & \multirow{2}{*}{0.000} & \multirow{2}{*}{ HS } \\
\hline & Range & $6.6-10.1$ & $6.7-14.3$ & $7-14.3$ & & & \\
\hline \multirow{2}{*}{ Serum iron $(\mu \mathrm{g} / \mathrm{mL})$} & Mean \pm SD & $65.33 \pm 20.36$ & $66.38 \pm 18.88$ & $53.06 \pm 22.16$ & \multirow{2}{*}{$4.159^{b}$} & \multirow{2}{*}{0.019} & \multirow{2}{*}{$\mathrm{S}$} \\
\hline & Range & $28-94$ & 27-109 & 16-98 & & & \\
\hline \multirow{2}{*}{ TIBC } & Mean \pm SD & $185.46 \pm 47.19$ & $204.94 \pm 58.36$ & $216.56 \pm 77.32$ & \multirow{2}{*}{$1.676^{\mathrm{b}}$} & \multirow{2}{*}{0.193} & \multirow{2}{*}{ NS } \\
\hline & Range & 105-303 & $112-311$ & $112-390$ & & & \\
\hline \multirow{2}{*}{ Ferritin (ng/mL) } & Mean \pm SD & $471.54 \pm 139.92$ & $399.62 \pm 158.78$ & $374.94 \pm 216.51$ & \multirow{2}{*}{$2.131^{b}$} & (125 & $N C$ \\
\hline & Range & $236-764$ & 15.9-720 & 16-782 & & 0.120 & No \\
\hline TSAT (\%) & Mean \pm SD & $35.16 \pm 8.12$ & $33.12 \pm 7.90$ & $26.87 \pm 14.66$ & $4670^{b}$ & 0012 & $\mathrm{~s}$ \\
\hline & Range & $9-47$ & $8-46$ & 5-56 & & & \\
\hline Corum albumin (otd) & Mean \pm SD & $3.93 \pm 0.39$ & $3.94 \pm 0.31$ & $3.84 \pm 0.48$ & $0610 \mathrm{~b}$ & 0.541 & $\Delta \mathrm{C}_{\mathrm{N}} \mathrm{C}$ \\
\hline ( & Range & $3.4-4.9$ & $3.5-4.7$ & $3.1-5.1$ & 0.010 & 0.041 & NO \\
\hline
\end{tabular}

$P$ value $>0.05$ : Non-significant (NS); $P$ value $<0.05$ : Significant $(S) ; P$ value $<0.01$ : Highly significant $(\mathrm{HS})$.

${ }^{a}$ Chi-square test; ${ }^{\mathrm{b}}$ One Way ANOVA test; ${ }^{\mathrm{c}}$ Kruskal-Wallis test.

However, there was no difference in prevalence of vitamin $\mathrm{D}$ deficiency between CKD patients and the general population in a cross sectional study by Guesseous et al (18). Low 25-hydroxy vitamin D levels have been associated with secondary hyperparathyroidism, decreased bone mineral density and high bone turnover in CKD and patients receiving chronic dialysis treatment $(19,20)$. Moreover, it has been associated with a decrease in muscle power and an increased fall risk (21). It has been

Table 5. Correlation of vitamin D3 levels with the other studied parameters using spearman correlation coefficient

\begin{tabular}{lcc}
\hline & \multicolumn{2}{c}{ Vitamin D3 } \\
\cline { 2 - 3 } & $\mathbf{R}$ & $\boldsymbol{P}$ value \\
\hline Age & -0.027 & 0.798 \\
Disease duration & -0.064 & 0.550 \\
Hemoglobin & $\mathbf{0 . 5 6 9 * *}$ & $\mathbf{0 . 0 0 0}$ \\
Serum calcium & $\mathbf{0 . 5 3 7 * *}$ & $\mathbf{0 . 0 0 0}$ \\
Serum phosphorus & 0.173 & 0.103 \\
Intact PTH & $\mathbf{- 0 . 7 3 3 ^ { * * }}$ & $\mathbf{0 . 0 0 0}$ \\
Serum iron & -0.197 & 0.062 \\
TIBC & 0.184 & 0.082 \\
Ferritin & -0.180 & 0.090 \\
TSAT (\%) & $\mathbf{- 0 . 3 0 5 * *}$ & $\mathbf{0 . 0 0 3}$ \\
Serum albumin & -0.129 & 0.226 \\
\hline
\end{tabular}

$P$ value $<0.05$ : Significant; $P$ value $<0.01$ : Highly significant. suggested in studies conducted on bone marrow red cell precursor cells that the active form of $25 \mathrm{OH}$ vitamin $\mathrm{D}$, calcitriol is associated with upregulating erythropoietinreceptors. Additionally, the active form of $25 \mathrm{OH}$ vitamin $\mathrm{D}$ also increases cell membrane permeability to calcium stimulating and proliferation of pre-erythroid cell series along with erythropoietin (22).

Our study is cross-sectional that recruited 90 ESRD patients on regular HD in Al Agouza hospital HD center.

In the current study, $66 \%$ of patients had vitamin D levels below $30 \mathrm{ng} / \mathrm{mL}$. Several studies have shown a high prevalence of vitamin D deficiency and insufficiency in CKD patients sometimes reaching over $80 \%$ (23). There are various causes of $25(\mathrm{OH})$ vitamin $\mathrm{D}$ deficiency in dialysis and CKD population like deficient sunlight exposure (24), impaired vitamin D synthesis and metabolism (25), uremic gastritis and dietary restrictions (26).

In our study, we divided our participants according to their vitamin D status into deficient, insufficient and sufficient groups. We found a highly significant relation between $25(\mathrm{OH})$ vitamin D levels and hemoglobin levels of our patients $(P<0.001)$. The patients with sufficient $25(\mathrm{OH})$ vitamin $\mathrm{D}$ levels had higher mean hemoglobin levels than those with insufficient $25(\mathrm{OH})$ vitamin $\mathrm{D}$ levels and subsequently than those with deficient $25(\mathrm{OH})$ vitamin D levels (mean 11.24 $\pm 1.83,9.24 \pm 1.43$, and 8.73 $\pm 0.83 \mathrm{ng} / \mathrm{dL}$, respectively). Similarly, Patel et al, found 
Table 6. Relationship between vitamin D3 levels in all patients and the other demographic parameters

\begin{tabular}{|c|c|c|c|c|c|c|}
\hline & & \multicolumn{2}{|l|}{ Vitamin D3 } & \multirow{2}{*}{ - Test value } & \multirow{2}{*}{$P$ value } & \multirow{2}{*}{ Sig } \\
\hline & & Median (IQR) & Range & & & \\
\hline \multirow{2}{*}{ Gender } & Female & $15.95(11-32)$ & $3.8-51$ & \multirow{2}{*}{$-0.521^{\mathrm{a}}$} & \multirow{2}{*}{0.602} & \multirow{2}{*}{ NS } \\
\hline & Male & $16.85(8-34.5)$ & $3.5-66.3$ & & & \\
\hline \multirow{2}{*}{ Diabetic } & No & $19.5(10-36)$ & $4-66.3$ & \multirow{2}{*}{$-1.691^{a}$} & \multirow{2}{*}{0.091} & \multirow{2}{*}{ NS } \\
\hline & Yes & $14.3(6.8-24.5)$ & $3.5-57$ & & & \\
\hline \multirow{2}{*}{ HTN } & No & $35.2(25.35-41.7)$ & $17.7-46$ & \multirow{2}{*}{$-1.831^{\mathrm{a}}$} & \multirow{2}{*}{0.067} & \multirow{2}{*}{ NS } \\
\hline & Yes & $16(8-33.8)$ & $3.5-66.3$ & & & \\
\hline \multirow{9}{*}{ Etiology of ESRD } & Hypertension & $30.7(8-37)$ & $4-51$ & \multirow{9}{*}{$9.119^{b}$} & \multirow{9}{*}{0.332} & \multirow{9}{*}{ NS } \\
\hline & Diabetic nephropathy & $14.3(6.8-24.5)$ & $3.5-57.0$ & & & \\
\hline & Lupus Nephritis & $13.4(9.25-24.9)$ & $5.5-36$ & & & \\
\hline & Chronic glomerulonephritis & $32(31.5-35.5)$ & 31-39 & & & \\
\hline & Chronic tubulointerstitial nephritis & $17.85(17.7-18)$ & $17.7-18$ & & & \\
\hline & ADPKD & $13(13-13)$ & 13-13 & & & \\
\hline & Hereditary nephritis & $46(46-46)$ & $46-46$ & & & \\
\hline & Amyloidosis & $37.4(37.4-37.4)$ & $37.4-37.4$ & & & \\
\hline & Unknown & $12.65(9-18.85)$ & $6.7-66.3$ & & & \\
\hline \multirow{3}{*}{ Dialysis access } & Arteriovenous fistula & $16(8-34)$ & $3.5-66.3$ & \multirow{3}{*}{$0.230^{\mathrm{b}}$} & \multirow{3}{*}{0.891} & \multirow{3}{*}{ NS } \\
\hline & Arteriovenous graft & $22(8.3-34)$ & $8.3-34$ & & & \\
\hline & Permanent HD catheter & $20(18-22)$ & $18-22$ & & & \\
\hline
\end{tabular}

ESRD, end-stage renal disease; ADPKD, autosomal dominant polycystic kidney disease; HD, hemodialysis.

$P$ value $>0.05$ : Non-significant $(\mathrm{NS}) ; P$ value $<0.05$ : Significant $(\mathrm{S}) ; P$ value $<0.01$ : Highly significant $(\mathrm{HS})$.

${ }^{\text {a }}$ Mann-Whitney test; ${ }^{\mathrm{b}}$ Kruskal-Wallis test.

Table 7. Hemoglobin level in relation to demographics data and vitamin D status

\begin{tabular}{|c|c|c|c|c|c|c|}
\hline & & \multicolumn{2}{|l|}{ Hemoglobin } & \multirow{2}{*}{ Test value } & \multirow{2}{*}{$P$ value } & \multirow{2}{*}{ Sig. } \\
\hline & & Mean \pm SD & Range & & & \\
\hline \multirow{2}{*}{ Gender } & Female & $9.38 \pm 1.62$ & $6.6-12.3$ & \multirow{2}{*}{$-1.431 \bullet$} & \multirow{2}{*}{0.156} & \multirow{2}{*}{ NS } \\
\hline & Male & $10.02 \pm 1.88$ & $7-14.3$ & & & \\
\hline \multirow{2}{*}{ Diabetic } & No & $9.95 \pm 1.92$ & $6.6-14$ & \multirow{2}{*}{$0.626 \bullet$} & \multirow{2}{*}{0.533} & \multirow{2}{*}{ NS } \\
\hline & Yes & $9.7 \pm 1.68$ & $7.9-14.3$ & & & \\
\hline \multirow{2}{*}{ HTN } & No & $10.15 \pm 2.22$ & $7.6-12.3$ & \multirow{2}{*}{$0.322 \bullet$} & \multirow{2}{*}{0.748} & \multirow{2}{*}{ NS } \\
\hline & Yes & $9.85 \pm 1.83$ & $6.6-14.3$ & & & \\
\hline \multirow{9}{*}{$\begin{array}{l}\text { Etiology } \\
\text { of ESRD }\end{array}$} & Hypertension & $10.19 \pm 2.07$ & $6.6-14$ & \multirow{9}{*}{$1.213 \bullet \bullet$} & \multirow{9}{*}{0.302} & \multirow{9}{*}{ NS } \\
\hline & Diabetic nephropathy & $9.69 \pm 1.68$ & $7.9-14.3$ & & & \\
\hline & Lupus nephritis & $8.78 \pm 1.44$ & $6.7-10$ & & & \\
\hline & Chronic glomerulonephritis & $11 \pm 1.73$ & $8.4-11.9$ & & & \\
\hline & Chronic tubulointerstitial nephritis & $8 \pm 0.57$ & $7.6-8.4$ & & & \\
\hline & ADPKD & $8.5 \pm 0$ & $8.5-8.5$ & & & \\
\hline & Hereditary nephritis & $9 \pm 0$ & $9-9$ & & & \\
\hline & Amyloidosis & $12.3 \pm 0$ & $12.3-12.3$ & & & \\
\hline & Unknown & $9.4 \pm 1.17$ & $8.2-11.9$ & & & \\
\hline \multirow{3}{*}{ Dialysis access } & Av fistula & $9.86 \pm 1.86$ & $6.6-14.3$ & \multirow{3}{*}{$0.041 \bullet \bullet$} & \multirow{3}{*}{0.960} & \multirow{3}{*}{ NS } \\
\hline & Av graft & $9.67 \pm 1$ & $8.7-10.7$ & & & \\
\hline & Permanent HD catheter & $10.15 \pm 2.47$ & $8.4-11.9$ & & & \\
\hline \multirow{3}{*}{$\begin{array}{l}\text { Vitamin D3 } \\
\text { (ng/dL) }\end{array}$} & Deficient & $8.73 \pm 0.83$ & $6.6-10.1$ & \multirow{3}{*}{24.660} & \multirow{3}{*}{0.000} & \multirow{3}{*}{$\mathrm{HS}$} \\
\hline & Insufficient & $9.24 \pm 1.43$ & $6.7-14.3$ & & & \\
\hline & Sufficient & $11.24 \pm 1.83$ & $7-14.3$ & & & \\
\hline
\end{tabular}

ESRD, end-stage renal disease; ADPKD, autosomal dominant polycystic kidney disease; HD, hemodialysis.

$P$ value $>0.05$ : Non-significant (NS); $P$ value $<0.05$ : Significant $(\mathrm{S}) ; P$ value $<0.01$ : Highly significant $(\mathrm{HS})$.

that $25(\mathrm{OH})$ vitamin $\mathrm{D}$ deficiencies were significantly associated with anemia in patients with early CKD (14). However, most patients in that study were CKD stage 3 and none were on dialysis.

Furthermore, it was clear that higher levels of vitamin
D were significantly associated with lower PTH levels. Serum PTH levels in patients with deficient and insufficient $25(\mathrm{OH})$ vitamin D levels were higher than those with sufficient $25(\mathrm{OH})$ vitamin D levels with median values $(669.5,528.5$ and $157.5 \mathrm{pg} / \mathrm{mL}$ respectively; 
Table 8. Correlation of hemoglobin level with the other parameters using Spearman's correlation coefficient

\begin{tabular}{lcc}
\hline & \multicolumn{2}{c}{ Hemoglobin } \\
\cline { 2 - 3 } & R & P value \\
\hline Vitamin D3 & $0.569^{* *}$ & 0.000 \\
Age & 0.033 & 0.758 \\
Disease duration & -0.171 & 0.107 \\
Serum calcium $(\mathrm{mg} / \mathrm{dL})$ & $0.410^{* *}$ & 0.000 \\
Serum phosphorus $(\mathrm{mg} / \mathrm{dL})$ & 0.109 & 0.308 \\
PTH $(\mathrm{pg} / \mathrm{mL})$ & $-0.477^{* *}$ & 0.000 \\
Serum iron $(\mu \mathrm{g} / \mathrm{mL})$ & -0.004 & 0.972 \\
TIBC & 0.022 & 0.835 \\
Ferritin $(\mathrm{ng} / \mathrm{mL})$ & 0.047 & 0.663 \\
T.SAT $(\%)$ & -0.005 & 0.962 \\
Serum albumin $(\mathrm{g} / \mathrm{dL})$ & -0.198 & 0.061 \\
\hline
\end{tabular}

$P<0.001)$. Similarly, several studies detected also negative correlation between $25(\mathrm{OH})$ vitamin $\mathrm{D}$ and serum PTH levels, establishing the role of vitamin $\mathrm{D}$ in suppressing secondary hyperparathyroidism along with calcitriol $(19,20)$.

High PTH levels were significantly associated with lower hemoglobin levels in our study. Bone marrow fibrosis can occur in high PTH levels leading to both anemia and erythropoietin resistance in CKD and dialysis patients (27). However, in the studies that examined the relation between hyperparathyroidism and anemia, 25(OH) vitamin D levels were not routinely measured (28).

Vitamin D has an anti-inflammatory action, reducing interleukin 6 and hepcidin levels leading to improved erythropoietin hyporesponsiveness (29). In our study, ferritin levels were higher in patients with deficient levels of vitamin D, however, this was not found to be statistically significant. A significant negative relation between vitamin D level and serum iron $(P=0.019)$ and transferrin saturation $(\%)(P=0.012)$ was clear in our study. Patients with vitamin $D$ deficiency had higher serum iron level and higher transferrin saturation levels compared with those with sufficient vitamin D levels. This finding, together with higher levels of ferritin in patients with deficient vitamin D may suggest that inflammation may play a role in the pathogenesis of anemia in those patients. Our study also supports the findings of Patel et al who found no evidence of iron deficiency in vitamin $\mathrm{D}$ deficient $\mathrm{CKD}$ patients (14).

Anemia was defined as hemoglobin level less than $11 \mathrm{~g} / \mathrm{dL}$. Risk factors associated with lower hemoglobin levels in this study included deficient vitamin D levels, high parathyroid hormone levels and high calcium levels. There was no statistically significant association between hemoglobin and duration of dialysis, age of patients, original kidney disease and type of vascular access. However, several studies including Madore et al have found age, race, gender erythropoietin (EPO) dose and frequency that EPO was administered, iron status (serum iron, transferrin saturation and ferritin), serum albumin, and the efficiency of dialysis by urea reduction ratio, were all found to be significantly associated with hemoglobin concentration (30). In the current study, all patients were on erythropoietin twice weekly, except those with hemoglobin over $11 \mathrm{~g} / \mathrm{dL}$, during the time of the study.

\section{Conclusion}

This study has found a statistically significant relation between the patients' vitamin D status and hemoglobin concentration. There was a correlation between vitamin D deficiency and anemia in our studied population. Further studies are needed to establish a causal relationship between 25 hydroxy vitamin D deficiency and anemia.

\section{Limitations of the study}

A few limitations should be noted. First, this was a single-center study; hence, additional studies with larger numbers of participants are needed to further establish the relationship between vitamin $\mathrm{D}$ deficiency and anemia. Second, it is difficult to establish a causal relationship between vitamin $\mathrm{D}$ deficiency and anemia as the current cross-sectional study design. Lastly more factors related to anemia in ESRD were not available as C-reactive protein, hepcidin and dialysis adequacy.

\section{Authors' contribution}

EN and LK conceived the idea, AT and ER contributed to the data collection and analysis. LK wrote the manuscript. All authors participated in preparing the final draft of the manuscript, revised the manuscript and critically evaluated the intellectual contents. All authors have read and approved the content of the manuscript and confirmed the accuracy or integrity of any part of the work.

\section{Ethical considerations}

Ethical issues (including plagiarism, data fabrication, double publication) have been completely observed by the authors.

\section{Conflicts of interest}

The authors declare that they have no competing interests.

\section{Funding/Support}

No funding was provided for this study.

\section{References}

1. Kazmi WH, Kausz AT, Khan S, Abichandani R, Ruthazer R, Obrador GT, et al Anemia: an early complication of chronic renal insufficiency. Am J Kidney Dis. 2001;38(4):803-12.

2. Horwich TB, Fonarow GC, Hamilton MA, MacLellan WR, Borenstein J. Anemia is associated with worse symptoms, greater impairment in functional capacity and a significant increase in mortality in patients with advanced heart failure. J Am Coll Cardiol. 2002;39:1780-6.

3. Walker AM, Schneider G, Yeaw J, Nordstrom B, Robbins S, Pettitt D. Anemia as a predictor of cardiovascular events in patients with elevated serum creatinine. J Am Soc Nephrol. 2006; 17:2293-8

4. Abramson JL, Jurkovitz CT, Vaccarino V, Weintraub WS, McClellan W. Chronic kidney disease, anemia, and incident stroke in a middle-aged, community-based population: the ARIC Study. Kidney Int. 2003;64:610-5.

5. Agarwal AK. Practical approach to the diagnosis and treatment 
of anemia associated with CKD in elderly. J Am Med Dir Assoc. 2006;7:S7-S12; quiz S17-21.

6. Jean G, Souberbielle JC, Chazot C. Vitamin D in Chronic Kidney Disease and Dialysis Patients. Nutrients. 2017;9(4):328.

7. Del Valle E, Negri AL, Aguirre C, Fradinger E, Zanchetta JR. Prevalence of $25(\mathrm{OH})$ vitamin D insufficiency and deficiency in chronic kidney disease stage 5 patients on hemodialysis. Hemodial Int. 2007;11:315-21.

8. Franca Gois PH, Wolley M, Ranganathan D, Seguro AC. Vitamin D Deficiency in Chronic Kidney Disease: Recent Evidence and Controversies. Int J Environ Res Public Health. 2018;15:1773.

9. Kolla PK, Desai M, Pathapati RM, Mastan Valli B, Pentyala S, Madhusudhan Reddy G, et al. Cutaneous manifestations in patients with chronic kidney disease on maintenance hemodialysis. ISRN Dermatol. 2012;2012:679619.

10. Holick M.F. Vitamin D deficiency. N Engl J Med. 2007; 357:266-81.

11. Bhan I, Dobens D., Tamez H, Deferio JJ, Li YC, Warren HS, et al. Nutritional vitamin D supplementation in dialysis: A randomized trial. Clin J Am Soc Nephrol. 2015;10:611-9.

12. Melamed ML, Michos ED, Post W, Astor B. 25-hydroxyvitamin $D$ levels and the risk of mortality in the general population. Arch Intern Med. 2008;168:1629-37. doi: 10.1001/ archinte.168.15.1629.

13. Shaffi K, Tighiouart H, Scott T, Lou K, Drew D, Weiner D, et al. Low 25-hydroxyvitamin D levels and cognitive impairment in hemodialysis patients. Clin J Am Soc Nephrol. 2013;8:979-86. doi: 10.2215/CJN.10651012.

14. Patel NM, Gutiérrez OM, Andress DL, Coyne DW, Levin A, Wolf M. Vitamin D deficiency and anemia in early chronic kidney disease. Kidney Int. 2010;77:715-20.

15. KendrickJ, Targher G, Smits G, Chonchol M.25-Hydroxyvitamin $\mathrm{D}$ deficiency and inflammation and their association with hemoglobin levels in chronic kidney disease. Am J Nephrol. 2009;30:64-72.

16. Coyne DW. The KDOQI US commentary on KDIGO anemia guideline and quality of life. Am J Kidney Dis. 2014;63:540

17. Kim SM, Choi HJ, Lee JP, Kim DK, Oh YK, Kim YS, et al. Prevalence of vitamin D deficiency and effects of supplementation with cholecalciferol in patients with chronic kidney disease. J Ren Nutr. 2014;24:20-5.

18. Guessous I, McClellan W, Kleinbaum D, Vaccarino V, Zoller O, Theler JM, et al . Swiss Survey on Salt Group. Comparisons of serum vitamin D levels, status, and determinants in populations with and without chronic kidney disease not requiring renal dialysis: a 24-hour urine collection population-based study. J
Ren Nutr. 2014;24:303-12.

19. Mucsi I, Almási C, Deák G, Marton A, Ambrus C, Berta K, et al. Serum 25(OH)-vitamin D levels and bone metabolism in patients on maintenance hemodialysis. Clin Nephrol. 2005;64:288-94

20. Milinković NLJ, Majkić-Singh NT, Mirković DD, Beletić AD, Pejanović SD, Vujanić ST. Relation between 25(OH)-vitamin D deficiency and markers of bone formation and resorption in haemodialysis patients. Clin Lab. 2009;55:333-9.

21. Boudville N, Inderjeeth C, Elder GJ, Glendenning P. Association between 25-hydroxyvitamin $\mathrm{D}$, somatic muscle weakness and falls risk in end-stage renal failure. Clin Endocrinol (Oxf). 2010;73:299-304

22. Alon DB, Chaimovitz C, Dvilansky A, Lugassy G, Douvdevani A, Shany S, et al . Novel role of $1,25(\mathrm{OH})(2) \mathrm{D}(3)$ in induction of erythroid progenitor cell proliferation. Exp Hematol. 2002;30:403-9.

23. Ngai $\mathrm{M}$, Lin $\mathrm{V}$, Wong HC, Vathsala A, How P. Vitamin D status and its association with mineral and bone disorder in a multiethnic chronic kidney disease population. Clin Nephrol. 2014;82:231-9.

24. Del Valle E, Negri AL, Aguirre C, Fradinger E, Zanchetta JR. Prevalence of $25(\mathrm{OH})$ vitamin D insufficiency and deficiency in chronic kidney disease stage 5 patients on hemodialysis. Hemodial Int. 2007;11:315-21.

25. Cuppari L, Garcia-Lopes MG. Hypovitaminosis D in Chronic Kidney Disease Patients: Prevalence and Treatment. J Ren Nutr. 2009;19:38-43. doi: 10.1053/j.jrn.2008.10.005.

26. Vaziri ND, Hollander D, Hung EK, Vo M, Dadufalza L. Impaired intestinal absorption of vitamin D3 in azotemic rats. Am J Clin Nutr. 1983;37:403-6.

27. Neves PL, Triviño J, Casaubon F, Santos V, Mendes P, Romão $P$, et al. Elderly patients on chronic hemodialysis with hyperparathyroidism: increase of hemoglobin level after intravenous calcitriol. Int Urol Nephrol. 2006;38:175-7.

28. Kalantar-Zadeh K, Lee GH, Miller JE, Streja E, Jing J, Robertson JA, et al. Predictors of hyporesponsiveness to erythropoiesisstimulating agents in hemodialysis patients. Am J Kidney Dis. 2009;53:823-34.

29. Türk S, Akbulut $M$, Yildiz A, Gürbilek $M$, Gönen $S$, Tombul $Z$, et al. Comparative effect of oral pulse and intravenous calcitriol treatment in hemodialysis patients: the effect on serum IL-1 and IL-6 levels and bone mineral density. Nephron. 2002;90:188-94.

30. Madore F, Lowrie EG, Brugnara C, Lew NL, Lazarus JM, Bridges $\mathrm{K}$, et al. Anemia in hemodialysis patients: variables affecting this outcome predictor. J Am Soc Nephrol. 1997;8:1921-9. 